\title{
M/M/1 Model with Unreliable Service
}

\author{
Joshua Patterson $^{1} \&$ Andrzej Korzeniowski ${ }^{1}$ \\ ${ }^{1}$ Department of Mathematics, University of Texas at Arlington, Arlington TX, USA \\ Correspondence: Joshua Patterson, Department of Mathematics, University of Texas at Arlington, Arlington TX, 76019, \\ USA. E-mail: Joshua.Patterson@uta.edu
}

Received: December 1, 2017 Accepted: December 22, 2017 Online Published: December 29, 2017

doi:10.5539/ijsp.v7n1p125

URL: https://doi.org/10.5539/ijsp.v7n1p125

\begin{abstract}
We define a new term unreliable service and construct the corresponding embedded Markov Chain to an $\mathrm{M} / \mathrm{M} / 1$ queue with so defined protocol. Sufficient conditions for positive recurrence and closed form of stationary distribution are provided. Furthermore, we compute the probability generating function of the stationary queue length and LaplaceStieltjes transform of the stationary waiting time. In the course of the analysis an interesting decomposition of both the queue length and waiting time has emerged. A number of queueing models can be recovered from our work by taking limits of certain parameters.
\end{abstract}

Keywords: unreliable service, quasi birth death process, matrix-geometric, stochastic decomposition

\section{Introduction}

Queueing theory covers a large body of models including queues with interruptions, breakdowns, batch arrivals, batch service, and the like. A typical assumption made for these models reads: at the moment of service time completion, the customer is assumed to have been served. This, however, need not be the case! Imagine for example that a service representative (server) has a message to communicate (in audible words) to an arriving client (customer) in a loud noisy environment. The customer is expected to hear the message spoken by the server but due to the noisy environment, the service may fail. That is, the person who was supposed to hear the message could not fully understand the message. Under this scenario, it was not the servers fault but instead the external interference that caused the service to fail. This is the prevailing idea which motivated our work in this paper.

Specifically, we seek to consider and analyze an M/M/1 queue with what we call 'unreliable service.' In this model, the term 'unreliable service' refers to the fact that the server may not always complete its service successfully. While service failure has been studied extensively in the literature, our model is different in that the failure is not due to the server itself by means of a 'breakdown,' nor is it due to the customer leaving the queue during the service time. Rather, the success or failure of a job is due to external forces and entirely random. Furthermore, neither the customer nor server know whether a job has failed or was successful until after the job's service time has been completed. The application of such a queue can come from many different areas and fields - all that is necessary is for some sort of quality check to be performed after service. This quality check would look at some set of measurements with certain thresholds and would conclude that the service was either successful or not.

Another key aspect to our model is that it will preserve the FCFS (First Come First Serve) discipline structure of the queue. Namely, when a customer's service fails, the customer does not lose its place in the queue and the service is repeated until it is successful. Our approach utilizes an embedded Markov chain methodology, similar to that done by Xu, Xiuli and Tian, Naishuo (Xu \& Tian, 2009). It should be noted that one can construct an M/PH/1 queue with similar properties. However, such a model will impose an additional, undesirable restriction: $\mu \geqslant \beta_{1}+\beta_{2}$ (Latouche \& Ramaswami, 1999) See below for definitions of these parameters.

\section{Definitions}

We begin by defining our process, state space, and parameters.

Definition 2.1. Let $\{N(t) \mid t \geqslant 0\}$ be the number of customers in the queue at time $t$, and

$S(t)= \begin{cases}1 & \text { immediately after service is rendered } \\ 0 & \text { otherwise }\end{cases}$

Then $\{(\mathrm{N}(\mathrm{t}), \mathrm{S}(\mathrm{t})) \mid \mathrm{t} \geqslant 0\}$ is a Markov process on the state space:

$\Omega=\{(0,0)\} \cup\{(k, s) \mid k \in \mathbb{N}, s \in\{0,1\}\}$

Define the following parameters: 
- $\lambda$ : the rate of the Poisson arrivals process.

- $\mu$ : the rate of service, successful or not.

- $\beta_{1}$ : the rate of a successful service.

- $\beta_{2}$ : the rate of a failed service.

To visualize such a Markovian process, it is helpful to construct the state transition rate diagram.

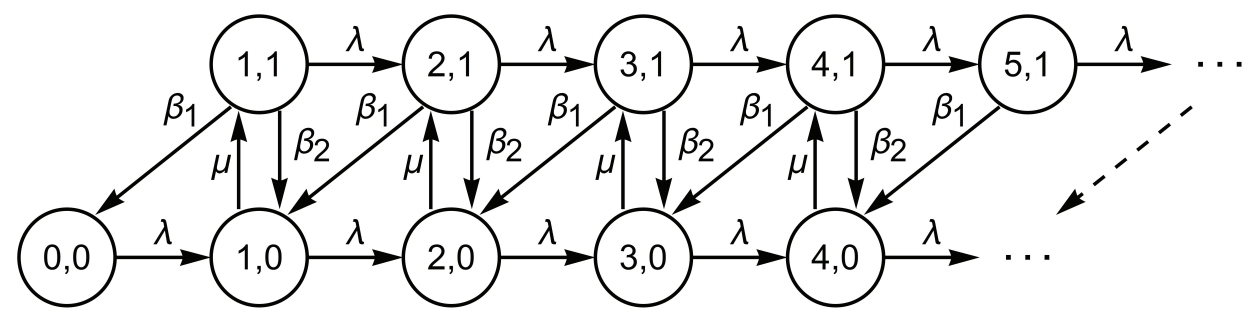

Figure 1. Markovian state transition rate diagram.

Formally, we define a 'successful service' to be a transition from $(n, 1) \longrightarrow(n-1,0)$, which is represented in the state transition diagram as having rate $\beta_{1}$. Similarly, we define a 'failed service' to be a transition from $(n, 1) \longrightarrow(n, 0)$ with transition rate $\beta_{2}$. Accordingly, we can compute the probabilities of a 'successful' or 'failed' service explicitly by considering the transition probabilities of the embedded Markov Chain.

$$
\begin{array}{ll}
p_{s}=\frac{\beta_{1}}{\beta_{1}+\beta_{2}+\lambda} \sum_{i=0}^{\infty}\left(\frac{\lambda}{\beta_{1}+\beta_{2}+\lambda}\right)^{i} & p_{f}=\frac{\beta_{2}}{\beta_{1}+\beta_{2}+\lambda} \sum_{i=0}^{\infty}\left(\frac{\lambda}{\beta_{1}+\beta_{2}+\lambda}\right)^{i} \\
p_{s}=\frac{\beta_{1}}{\beta_{1}+\beta_{2}+\lambda}\left(\frac{1}{1-\frac{\lambda}{\beta_{1}+\beta_{2}+\lambda}}\right) & p_{f}=\frac{\beta_{2}}{\beta_{1}+\beta_{2}+\lambda}\left(\frac{1}{1-\frac{\lambda}{\beta_{1}+\beta_{2}+\lambda}}\right) \\
p_{s}=\frac{\beta_{1}}{\beta_{1}+\beta_{2}+\lambda}\left(\frac{1}{\left.\frac{\beta_{1}+\beta_{2}}{\beta_{1}+\beta_{2}+\lambda}\right)}\right) & p_{f}=\frac{\beta_{2}}{\beta_{1}+\beta_{2}+\lambda}\left(\frac{1}{\frac{\beta_{1}+\beta_{2}}{\beta_{1}+\beta_{2}+\lambda}}\right) \\
p_{s}=\frac{\beta_{1}}{\beta_{1}+\beta_{2}+\lambda}\left(\frac{\beta_{1}+\beta_{2}+\lambda}{\beta_{1}+\beta_{2}}\right) & p_{f}=\frac{\beta_{2}}{\beta_{1}+\beta_{2}+\lambda}\left(\frac{\beta_{1}+\beta_{2}+\lambda}{\beta_{1}+\beta_{2}}\right) \\
p_{s}=\frac{\beta_{1}}{\beta_{1}+\beta_{2}} & p_{f}=\frac{\beta_{2}}{\beta_{1}+\beta_{2}}
\end{array}
$$

From here, we can list the countable state space in lexicographical order; formally defined below.

Definition 2.2. Lexicographical Ordering

We say $\left(k_{1}, s_{1}\right)<\left(k_{2}, s_{2}\right)$ if and only if $k_{1}<k_{2}$ or $\left(k_{1}=k_{2}\right.$ and $\left.s_{1}<s_{2}\right)$

Using this re-ordering convention (see pg. 353 of Ibe, Oliver 2013), we can write $\Omega$ as follows:

$\Omega=\{(0,0),(0,1),(1,0),(1,1),(2,0),(2,1), \ldots\}$. Furthermore, we can define the corresponding infinitesimal matrix $\mathrm{Q}$.

\section{Infinitesimal Matrix Q}

$$
\mathbf{Q}=\left[\begin{array}{cccccc}
\hat{\mathbf{A}} & \hat{\mathbf{C}} & 0 & 0 & 0 & \ldots \\
\hat{\mathbf{B}} & \mathbf{A} & \mathbf{C} & 0 & 0 & \ldots \\
0 & \mathbf{B} & \mathbf{A} & \mathbf{C} & 0 & \ldots \\
\vdots & \vdots & \vdots & \ddots & \vdots & \vdots
\end{array}\right]
$$

where

$$
\begin{array}{lll}
\hat{\mathbf{A}}=[-\lambda] & \hat{\mathbf{B}}=\left[\begin{array}{c}
0 \\
\beta_{1}
\end{array}\right] & \hat{\mathbf{C}}=\left[\begin{array}{ll}
\lambda & 0
\end{array}\right] \\
\mathbf{A}=\left[\begin{array}{cc}
-(\lambda+\mu) & \mu \\
\beta_{2} & -\left(\beta_{1}+\beta_{2}+\lambda\right)
\end{array}\right] & \mathbf{B}=\left[\begin{array}{cc}
0 & 0 \\
\beta_{1} & 0
\end{array}\right] & \mathbf{C}=\left[\begin{array}{ll}
\lambda & 0 \\
0 & \lambda
\end{array}\right]
\end{array}
$$




\section{Positive Recurrence}

Since the matrix Q has a block-tridiagonal structure, we have a QBD (Quasi Birth Death) Markovian process. Accordingly, we apply Theorem 1.5.1 from Neuts (Neuts, 1981) to prove a lemma that will be used to show positive recurrence and find the stationary distribution explicitly. To this end, we need the following lemma.

Lemma 4.1. The irreducible, block-tridiagonal Markov process with infinitesimal matrix $\mathbf{Q}$ is positive recurrent if and only if:

- the minimal non-negative solution $\mathbf{R}$ of quadratic matrix equation:

$$
\mathbf{R}^{2} \mathbf{B}+\mathbf{R A}+\mathbf{C}=\mathbf{0}
$$

has $\operatorname{sp}(\mathbf{R})<1$, and

- there exists a positive vector $\left(\mathbf{x}_{\mathbf{0}}, \mathbf{x}_{\mathbf{1}}\right)$ such that $\left(\mathbf{x}_{\mathbf{0}}, \mathbf{x}_{\mathbf{1}}\right) \mathbf{B}[\mathbf{R}]=0$ where:

$\mathbf{B}[\mathbf{R}]=\left[\begin{array}{cc}\hat{\mathbf{A}} & \hat{\mathbf{C}} \\ \hat{\mathbf{B}} & \mathbf{A}+\mathbf{R B}\end{array}\right]$, and $\left(\mathbf{x}_{\mathbf{0}}, \mathbf{x}_{\mathbf{1}}\right)$ is normalized by $\mathbf{x}_{\mathbf{0}} \mathbf{e}+\mathbf{x}_{\mathbf{1}}(\mathbf{I}-\mathbf{R})^{-1} \mathbf{e}=\mathbf{1}$

The stationary distribution satisfying: $\left\{\begin{array}{ll}\pi \mathrm{Q}=\mathbf{0} \\ \pi \mathrm{e}=1\end{array}\right.$ is given by: $\pi_{\mathrm{k}}= \begin{cases}\mathbf{x}_{\mathbf{0}} & \text { if } \mathrm{k}=0 \\ \mathbf{x}_{\mathbf{1}} & \text { if } \mathrm{k}=1 \\ \mathbf{x}_{\mathbf{1}} \mathbf{R}^{\mathrm{k}-1} & \text { if } \mathrm{k} \geqslant 2\end{cases}$

Our lemma, unlike Theorem 1.5.1 (Neuts, 1981), is stated in terms of the infinitesimal matrix Q rather than a Markov chain transition probability matrix.

Proof. Let $\mathbf{P}=\mathbf{I}+\tau^{-1} \mathbf{Q}$, where $\tau=-\min \{\operatorname{diag}(\hat{\mathbf{A}}) \cup \operatorname{diag}(\mathbf{A})\}>0$. Then we have:

$$
\mathbf{P}=\left[\begin{array}{cccccc}
\hat{\mathbf{A}^{\prime}} & \hat{\mathbf{C}}^{\prime} & 0 & 0 & 0 & \ldots \\
\hat{\mathbf{B}}^{\prime} & \mathbf{A}^{\prime} & \mathbf{C}^{\prime} & 0 & 0 & \ldots \\
0 & \mathbf{B}^{\prime} & \mathbf{A}^{\prime} & \mathbf{C}^{\prime} & 0 & \ldots \\
\vdots & \vdots & \vdots & \ddots & \vdots & \vdots
\end{array}\right]=\left[\begin{array}{cccccc}
\mathbf{I}+\tau^{-1} \hat{\mathbf{A}} & \tau^{-1} \hat{\mathbf{C}} & 0 & 0 & 0 & \ldots \\
\tau^{-1} \hat{\mathbf{B}} & \mathbf{I}+\tau^{-1} \mathbf{A} & \tau^{-1} \mathbf{C} & 0 & 0 & \ldots \\
0 & \tau^{-1} \mathbf{B} & \mathbf{I}+\tau^{-1} \mathbf{A} & \tau^{-1} \mathbf{C} & 0 & \ldots \\
\vdots & \vdots & \vdots & \ddots & \vdots & \vdots
\end{array}\right]
$$

Since $\mathbf{P e}=\left(\mathbf{I}+\tau^{-1} \mathbf{Q}\right) \mathbf{e}=\mathbf{I e}=\mathbf{e}$

$\Longrightarrow \mathbf{P}$ is a stochastic probability matrix of a discrete time Markov chain.

Moreover, if $\pi \mathbf{P}=\pi \Longrightarrow \pi\left(\mathbf{I}+\tau^{-1} \mathbf{Q}\right)=\pi \Longrightarrow \pi \mathbf{Q}=0$

$$
\begin{aligned}
& \text { and } \pi \mathbf{Q}=0 \Longrightarrow \pi+\tau^{-1} \pi \mathbf{Q}=\boldsymbol{\pi} \Longrightarrow \boldsymbol{\pi}\left(\mathbf{I}+\tau^{-1} \mathbf{Q}\right)=\boldsymbol{\pi} \Longrightarrow \boldsymbol{\pi} \mathbf{P}=\boldsymbol{\pi} \\
& \Longrightarrow \pi \mathbf{Q}=0 \Longleftrightarrow \pi \mathbf{P}=1
\end{aligned}
$$

Theorem 1.5.1 from (Neuts, 1981) states that $\mathbf{P}$, and consequently $\mathbf{Q}$, is positive recurrent if and only if:

- the minimal non-negative solution $\mathbf{R}$ of quadratic matrix equation:

$$
\mathbf{R}^{2} \mathbf{B}^{\prime}+\mathbf{R} \mathbf{A}^{\prime}+\mathbf{C}^{\prime}=\mathbf{R}
$$

has $\operatorname{sp}(\mathbf{R})<1$, and

- there exists a positive vector $\left(\mathbf{x}_{\mathbf{0}}, \mathbf{x}_{\mathbf{1}}\right)$ such that $\left(\mathbf{x}_{\mathbf{0}}, \mathbf{x}_{\mathbf{1}}\right) \mathbf{B}^{\prime}[\mathbf{R}]=\left(\mathbf{x}_{\mathbf{0}}, \mathbf{x}_{\mathbf{1}}\right)$ where:

$\mathbf{B}^{\prime}[\mathbf{R}]=\left[\begin{array}{cc}\hat{\mathbf{A}}^{\prime} & \hat{\mathbf{C}}^{\prime} \\ \hat{\mathbf{B}}^{\prime} & \mathbf{A}^{\prime}+\mathbf{R B}^{\prime}\end{array}\right]$, and $\left(\mathbf{x}_{\mathbf{0}}, \mathbf{x}_{\mathbf{1}}\right)$ is normalized by $\mathbf{x}_{\mathbf{0}} \mathbf{e}+\mathbf{x}_{\mathbf{1}}(\mathbf{I}-\mathbf{R})^{-1} \mathbf{e}=\mathbf{1}$

The stationary distribution satisfying: $\left\{\begin{array}{l}\pi \mathbf{P}=\pi \\ \pi \boldsymbol{e}=1\end{array}\right.$ is given by: $\boldsymbol{\beta}_{\mathbf{k}}=\left\{\begin{array}{lll}\mathbf{x}_{\mathbf{0}} & \text { if } k=0 \\ \mathbf{x}_{\mathbf{1}} & \text { if } k=1 \\ \mathbf{x}_{\mathbf{1}} \mathbf{R}^{k-1} & \text { if } k \geqslant 2\end{array}\right.$

To finish our proof, we must restate the conditions on $\mathbf{P}$ in terms of conditions on $\mathbf{Q}$ : 


$$
\begin{aligned}
\mathbf{R}^{2} \mathbf{B}^{\prime}+\mathbf{R} \mathbf{A}^{\prime}+\mathbf{C}^{\prime}=\mathbf{R} & \Longleftrightarrow \tau^{-1} \mathbf{R}^{2} \mathbf{B}+\mathbf{R}\left(\mathbf{I}+\tau^{-1} \mathbf{A}\right)+\tau^{-1} \mathbf{C}=\mathbf{R} \\
& \Longleftrightarrow \tau^{-1} \mathbf{R}^{2} \mathbf{B}+\tau^{-1} \mathbf{R A}+\tau^{-1} \mathbf{C}=\mathbf{0} \\
& \Longleftrightarrow \mathbf{R}^{2} \mathbf{B}+\mathbf{R A}+\mathbf{C}=\mathbf{0}
\end{aligned}
$$

and

$$
\begin{aligned}
\left(\mathbf{x}_{\mathbf{0}}, \mathbf{x}_{\mathbf{1}}\right) \mathbf{B}^{\prime}[\mathbf{R}]=\left(\mathbf{x}_{\mathbf{0}}, \mathbf{x}_{\mathbf{1}}\right) & \Longleftrightarrow\left(\mathbf{x}_{\mathbf{0}}, \mathbf{x}_{\mathbf{1}}\right)\left[\begin{array}{cc}
\hat{\mathbf{A}}^{\prime} & \hat{\mathbf{C}}^{\prime} \\
\hat{\mathbf{B}}^{\prime} & \mathbf{A}^{\prime}+\mathbf{R} \mathbf{B}^{\prime}
\end{array}\right]=\left(\mathbf{x}_{\mathbf{0}}, \mathbf{x}_{\mathbf{1}}\right) \\
& \Longleftrightarrow\left(\mathbf{x}_{\mathbf{0}}, \mathbf{x}_{\mathbf{1}}\right)\left[\begin{array}{cc}
\mathbf{I}+\tau^{-1} \hat{\mathbf{A}} & \tau^{-1} \hat{\mathbf{C}} \\
\tau^{-1} \hat{\mathbf{B}} & \mathbf{I}+\tau^{-1}(\mathbf{A}+\mathbf{R B})
\end{array}\right]=\left(\mathbf{x}_{\mathbf{0}}, \mathbf{x}_{\mathbf{1}}\right) \\
& \Longleftrightarrow\left(\mathbf{x}_{\mathbf{0}}, \mathbf{x}_{\mathbf{1}}\right)\left(\mathbf{I}+\tau^{-1} \mathbf{B}[\mathbf{R}]\right)=\left(\mathbf{x}_{\mathbf{0}}, \mathbf{x}_{\mathbf{1}}\right) \\
& \Longleftrightarrow\left(\mathbf{x}_{\mathbf{0}}, \mathbf{x}_{\mathbf{1}}\right) \mathbf{B}[\mathbf{R}]=\mathbf{0}
\end{aligned}
$$

\section{The Quadratic Matrix Equation}

Thanks to Lemma 4.1, we seek the minimal non-negative solution $\mathbf{R}$ to the quadratic matrix equation:

$$
\mathbf{R}^{2} \mathbf{B}+\mathbf{R A}+\mathbf{C}=\mathbf{0}
$$

There are many methods for solving such equations in the literature. Some are numerical in nature (Guo, 2014), (S. Seo, J. Seo \& H. Kim, 2014), others are analytical for particular cases (Adan, Wessels, \& Zijm, 1993). However, pure analytical methods are generally preferred to numerical ones when they are feasible. In our case, we employ the direct method whereby we solve the system of equations generated by equating the matrices entry by entry.

Let $\mathbf{R}=\left[\begin{array}{ll}r_{11} & r_{12} \\ r_{21} & r_{22}\end{array}\right] \Longrightarrow$ (6) can be restated at the following system of equations:

$$
\left\{\begin{array}{l}
\lambda-(\lambda+\mu) r_{11}+\left(r_{11} r_{12}+r_{12} r_{22}\right) \beta_{1}+r_{12} \beta_{2}=0 \\
\mu r_{11}-r_{12}\left(\lambda+\beta_{1}+\beta_{2}\right)=0 \\
-(\lambda+\mu) r_{21}+\left(r_{12} r_{21}+r_{22}^{2}\right) \beta_{1}+r_{22} \beta_{2}=0 \\
\lambda+\mu r_{21}-r_{22}\left(\lambda+\beta_{1}+\beta_{2}\right)=0
\end{array}\right.
$$

The analytical minimal non-negative solution to $(7)$ is given by:

$$
\left\{\begin{array}{l}
r_{11}=\frac{\lambda\left(\lambda+\beta_{1}+\beta_{2}\right)}{\mu \beta_{1}} \\
r_{12}=\frac{\lambda}{\beta_{1}} \\
r_{21}=\frac{\lambda\left(\lambda+\beta_{2}\right)}{\mu \beta_{1}} \\
r_{22}=\frac{\lambda}{\beta_{1}}
\end{array} \quad \Longrightarrow \mathbf{R}=\left[\begin{array}{ll}
\frac{\lambda\left(\lambda+\beta_{1}+\beta_{2}\right)}{\mu \beta_{1}} & \frac{\lambda}{\beta_{1}} \\
\frac{\lambda\left(\lambda+\beta_{2}\right)}{\mu \beta_{1}} & \frac{\lambda}{\beta_{1}}
\end{array}\right]=\frac{\lambda}{\beta_{1}}\left[\begin{array}{cc}
\frac{\lambda+\beta_{1}+\beta_{2}}{\mu} & 1 \\
\frac{\lambda+\beta_{2}}{\mu} & 1
\end{array}\right]\right.
$$

\section{The Spectral Radius of $\mathbf{R}$}

At this point, we can compute the spectral radius of $\mathbf{R}$ explicitly and construct a more readily verifiable sufficient condition under which our model will be positive recurrent.

Corollary. By Lemma 4.1, the infinitesimal matrix $\mathbf{Q}$ given in equation (2) is positive recurrent if and only if: $\beta_{1}(\mu-\lambda)-\lambda\left(\mu+\beta_{2}\right)>0$.

Proof. We compute the spectral radius of $\mathbf{R}$ by solving the scalar quadratic equation generated by $\operatorname{det}\left(\mathbf{R}-\rho_{i} \mathbf{I}\right)=0$, yielding that $\rho_{i}$ satisfies the following quadratic equation:

$$
\begin{gathered}
\mu \beta_{1} \rho_{i}^{2}-\lambda\left(\lambda+\mu+\beta_{1}+\beta_{2}\right) \rho_{i}+\lambda^{2}=0 \\
\Longrightarrow \rho_{i}=\frac{\lambda\left(\lambda+\mu+\beta_{1}+\beta_{2}+(-1)^{i} \sqrt{\left(\lambda+\mu+\beta_{1}+\beta_{2}\right)^{2}-4 \mu \beta_{1}}\right)}{2 \mu \beta_{1}}, \quad i=1,2
\end{gathered}
$$

It is clear by inspection that the largest of these eigenvalues in (9) will contain the positive radical. Thus, by Lemma 4.1, $\mathbf{Q}$ is positive recurrent if and only if:

$\rho_{0}=\frac{\lambda\left(\lambda+\mu+\beta_{1}+\beta_{2}+\sqrt{\left(\lambda+\mu+\beta_{1}+\beta_{2}\right)^{2}-4 \mu \beta_{1}}\right)}{2 \mu \beta_{1}}<1$ 
$\Longleftrightarrow \lambda+\mu+\beta_{1}+\beta_{2}+\sqrt{\left(\lambda+\mu+\beta_{1}+\beta_{2}\right)^{2}-4 \mu \beta_{1}}<\frac{2 \mu \beta_{1}}{\lambda}$

$\Longleftrightarrow \sqrt{\left(\lambda+\mu+\beta_{1}+\beta_{2}\right)^{2}-4 \mu \beta_{1}}<\frac{2 \mu \beta_{1}}{\lambda}-\left(\lambda+\mu+\beta_{1}+\beta_{2}\right)$

$\Longleftrightarrow\left(\lambda+\mu+\beta_{1}+\beta_{2}\right)^{2}-4 \mu \beta_{1}<\frac{4 \mu^{2} \beta_{1}^{2}}{\lambda^{2}}-\frac{4 \mu \beta_{1}\left(\lambda+\mu+\beta_{1}+\beta_{2}\right)}{\lambda}+\left(\lambda+\mu+\beta_{1}+\beta_{2}\right)^{2}$

$\Longleftrightarrow-4 \mu \beta_{1}<\frac{4 \mu^{2} \beta_{1}^{2}}{\lambda^{2}}-\frac{4 \mu \beta_{1}\left(\lambda+\mu+\beta_{1}+\beta_{2}\right)}{\lambda}$

$\Longleftrightarrow-4 \mu \beta_{1} \lambda^{2}<4 \mu^{2} \beta_{1}^{2}-4 \mu \beta_{1} \lambda\left(\lambda+\mu+\beta_{1}+\beta_{2}\right)$

$\Longleftrightarrow 0<4 \mu \beta_{1} \lambda^{2}+4 \mu^{2} \beta_{1}^{2}-4 \lambda \mu \beta_{1}\left(\lambda+\mu+\beta_{1}+\beta_{2}\right)$

$\Longleftrightarrow 0<\lambda^{2}+\mu \beta_{1}-\lambda\left(\lambda+\mu+\beta_{1}+\beta_{2}\right)$

$\Longleftrightarrow 0<\mu \beta_{1}-\lambda\left(\mu+\beta_{1}+\beta_{2}\right)$

$\Longleftrightarrow \beta_{1}(\mu-\lambda)-\lambda\left(\mu+\beta_{2}\right)>0$

\section{The Stationary Distribution}

\subsection{The Explicit form of $\mathbf{R}^{\mathrm{k}}$}

Proposition 7.1. Using the scalar-factored form of $\mathbf{R}$ in (8), we find:

$$
\mathbf{R}^{k}=\left[\begin{array}{cc}
\frac{\left(\beta_{1} \rho_{0}-\lambda\right) \rho_{0}^{k}+\rho_{1}^{k}\left(\lambda-\beta_{1} \rho_{1}\right)}{\beta_{1}\left(\rho_{0}-\rho_{1}\right)} & \frac{\lambda\left(\rho_{0}^{k}-\rho_{1}^{k}\right)}{\beta_{1}\left(\rho_{0}-\rho_{1}\right)} \\
\frac{\lambda\left(\lambda+\beta_{2}\right)\left(\rho_{0}^{k}-\rho_{1}^{k}\right)}{\mu \beta_{1}\left(\rho_{0}-\rho_{1}\right)} & \frac{\left(\lambda-\beta_{1} \rho_{1}\right) \rho_{0}^{k}+\rho_{1}^{k}\left(\beta_{1} \rho_{0}-\lambda\right)}{\beta_{1}\left(\rho_{0}-\rho_{1}\right)}
\end{array}\right]
$$

Proof. By Mathematical Induction, we will show this is true for $k=1$, assume it is true for arbitrary $k$, then show it is true for $k+1$.

$\underline{\mathrm{k}=1}$

$$
\begin{aligned}
\mathbf{R}^{1} & =\left[\begin{array}{cc}
\frac{\left(\beta_{1} \rho_{0}-\lambda\right) \rho_{0}+\rho_{1}\left(\lambda-\beta_{1} \rho_{1}\right)}{\beta_{1}\left(\rho_{0}-\rho_{1}\right)} & \frac{\lambda\left(\rho_{0}-\rho_{1}\right)}{\beta_{1}\left(\rho_{0}-\rho_{1}\right)} \\
\frac{\lambda\left(\lambda+\beta_{2}\right)\left(\rho_{0}-\rho_{1}\right)}{\mu \beta_{1}\left(\rho_{0}-\rho_{1}\right)} & \frac{\left(\lambda-\beta_{1} \rho_{1}\right) \rho_{0}+\rho_{1}\left(\beta_{1} \rho_{0}-\lambda\right)}{\beta_{1}\left(\rho_{0}-\rho_{1}\right)}
\end{array}\right] \\
& =\left[\begin{array}{cc}
\frac{\beta_{1} \rho_{0}^{2}-\lambda \rho_{0}+\lambda \rho_{1}-\beta_{1} \rho_{1}^{2}}{\beta_{1}\left(\rho_{0}-\rho_{1}\right)} & \frac{\lambda}{\beta_{1}} \\
\frac{\lambda\left(\lambda+\beta_{2}\right)}{\mu \beta_{1}} & \frac{\lambda \rho_{0}-\beta_{1} \rho_{1} \rho_{0}+\beta_{1} \rho_{1} \rho_{0}-\lambda \rho_{1}}{\beta_{1}\left(\rho_{0}-\rho_{1}\right)}
\end{array}\right] \\
& =\left[\begin{array}{cc}
\frac{\beta_{1}\left(\rho_{0}^{2}-\rho_{1}^{2}\right)-\lambda \rho_{0}+\lambda \rho_{1}}{\beta_{1}\left(\rho_{0}-\rho_{1}\right)} & \frac{\lambda}{\beta_{1}} \\
\frac{\lambda\left(\lambda+\beta_{2}\right)}{\mu \beta_{1}} & \frac{\lambda \rho_{0}-\lambda \rho_{1}}{\beta_{1}\left(\rho_{0}-\rho_{1}\right)}
\end{array}\right] \\
& =\left[\begin{array}{cc}
\frac{\beta_{1}\left(\rho_{0}-\rho_{1}\right)\left(\rho_{0}+\rho_{1}\right)-\lambda\left(\rho_{0}-\rho_{1}\right)}{\beta_{1}\left(\rho_{0}-\rho_{1}\right)} & \frac{\lambda}{\beta_{1}} \\
\frac{\lambda\left(\lambda+\beta_{2}\right)}{\mu \beta_{1}} & \frac{\lambda\left(\rho_{0}-\rho_{1}\right)}{\beta_{1}\left(\rho_{0}-\rho_{1}\right)}
\end{array}\right] \\
& =\left[\begin{array}{cc}
\frac{\beta_{1}\left(\rho_{0}+\rho_{1}\right)-\lambda}{\beta_{1}} & \frac{\lambda}{\beta_{1}} \\
\frac{\lambda\left(\lambda+\beta_{2}\right)}{\mu \beta_{1}} & \frac{\lambda}{\beta_{1}}
\end{array}\right] \\
& =\left[\begin{array}{ll}
\frac{\lambda\left(\lambda+\beta_{1}+\beta_{2}\right)}{\mu \beta_{1}} & \frac{\lambda}{\beta_{1}} \\
\frac{\lambda\left(\lambda+\beta_{2}\right)}{\mu \beta_{1}} & \frac{\lambda}{\beta_{1}}
\end{array}\right],
\end{aligned}
$$

$\underline{\mathrm{k}+1}$

$$
\begin{aligned}
& \mathbf{R}^{\mathrm{k}} \mathbf{R}=\left[\begin{array}{cc}
\frac{\left(\beta_{1} \rho_{0}-\lambda\right) \rho_{0}^{k}+\rho_{1}^{k}\left(\lambda-\beta_{1} \rho_{1}\right)}{\beta_{1}\left(\rho_{0}-\rho_{1}\right)} & \frac{\lambda\left(\rho_{0}^{k}-\rho_{1}^{k}\right)}{\beta_{1}\left(\rho_{0}-\rho_{1}\right)} \\
\frac{\lambda\left(\lambda+\beta_{2}\right)\left(\rho_{0}^{k}-\rho_{1}^{k}\right)}{\mu \beta_{1}\left(\rho_{0}-\rho_{1}\right)} & \frac{\left(\lambda-\beta_{1} \rho_{1}\right) \rho_{0}^{k}+\rho_{1}^{k}\left(\beta_{1} \rho_{0}-\lambda\right)}{\beta_{1}\left(\rho_{0}-\rho_{1}\right)}
\end{array}\right]\left[\begin{array}{cc}
\frac{\lambda\left(\lambda+\beta_{1}+\beta_{2}\right)}{\mu \beta_{1}} & \frac{\lambda}{\beta_{1}} \\
\frac{\lambda\left(\lambda+\beta_{2}\right)}{\mu \beta_{1}} & \frac{\lambda}{\beta_{1}}
\end{array}\right] \\
& =\left[\begin{array}{cc}
\frac{\lambda\left(\lambda+\beta_{1}+\beta_{2}\right)\left(\left(\beta_{1} \rho_{0}-\lambda\right) \rho_{0}^{k}+\rho_{1}^{k}\left(\lambda-\beta_{1} \rho_{1}\right)\right)+\lambda^{2}\left(\lambda+\beta_{2}\right)\left(\rho_{0}^{k}-\rho_{1}^{k}\right)}{\mu \beta_{1}^{2}\left(\rho_{0}-\rho_{1}\right)} & \frac{\lambda}{\beta_{1}}\left(\frac{\left(\beta_{1} \rho_{0}-\lambda\right) \rho_{0}^{k}+\rho_{1}^{k}\left(\lambda-\beta_{1} \rho_{1}\right)+\lambda\left(\rho_{0}^{k}-\rho_{1}^{k}\right)}{\beta_{1}\left(\rho_{0}-\rho_{1}\right)}\right) \\
\frac{\lambda\left(\lambda+\beta_{2}\right)\left(\lambda\left(\lambda+\beta_{1}+\beta_{2}\right)\left(\rho_{0}^{k}-\rho_{1}^{k}\right)+\left(\lambda-\beta_{1} \rho_{1}\right) \mu \rho_{0}^{k}+\mu \rho_{1}^{k}\left(\beta_{1} \rho_{0}-\lambda\right)\right)}{\mu^{2} \beta_{1}^{2}\left(\rho_{0}-\rho_{1}\right)} & \frac{\lambda}{\beta_{1}}\left(\frac{\lambda\left(\lambda+\beta_{2}\right)\left(\rho_{0}^{k}-\rho_{1}^{k}\right)+\left(\lambda-\beta_{1} \rho_{1}\right) \mu \rho_{0}^{k}+\mu \rho_{1}^{k}\left(\beta_{1} \rho_{0}-\lambda\right)}{\mu \beta_{1}\left(\rho_{0}-\rho_{1}\right)}\right)
\end{array}\right]
\end{aligned}
$$

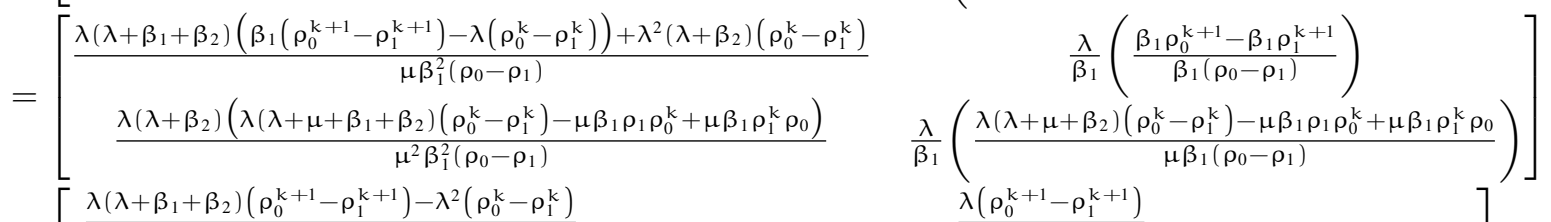

$$
\begin{aligned}
& =\left[\begin{array}{cc}
\frac{\lambda\left(\lambda+\beta_{1}+\beta_{2}\right)\left(\rho_{0}^{k+1}-\rho_{1}^{k+1}\right)-\lambda^{2}\left(\rho_{0}^{k}-\rho_{1}^{k}\right)}{\mu \beta_{1}\left(\rho_{0}-\rho_{1}\right)} & \frac{\lambda\left(\rho_{0}^{k+1}-\rho_{1}^{k+1}\right)}{\beta_{1}\left(\rho_{0}-\rho_{1}\right)} \\
\frac{\lambda\left(\lambda+\beta_{2}\right)\left(\left(\rho_{0}+\rho_{1}\right)\left(\rho_{0}^{k}-\rho_{1}^{k}\right)-\rho_{1} \rho_{0}^{k}+\rho_{1}^{k} \rho_{0}\right)}{\mu \beta_{1}\left(\rho_{0}-\rho_{1}\right)} & \frac{\lambda}{\beta_{1}}\left(\frac{\lambda\left(\lambda+\mu+\beta_{1}+\beta_{2}\right)\left(\rho_{0}^{k}-\rho_{1}^{k}\right)-\lambda \beta_{1}\left(\rho_{0}^{k}-\rho_{1}^{k}\right)-\mu \beta_{1} \rho_{1} \rho_{0}^{k}+\mu \beta_{1} \rho_{1}^{k} \rho_{0}}{\mu \beta_{1}\left(\rho_{0}-\rho_{1}\right)}\right)
\end{array}\right]
\end{aligned}
$$




$$
\begin{aligned}
& =\left[\begin{array}{cc}
\frac{\mu \beta_{1}\left(\rho_{0}+\rho_{1}\right)\left(\rho_{0}^{k+1}-\rho_{1}^{k+1}\right)-\lambda \mu\left(\rho_{0}^{k+1}-\rho_{1}^{k+1}\right)-\mu \beta_{1} \rho_{0} \rho_{1}\left(\rho_{0}^{k}-\rho_{1}^{k}\right)}{\mu \beta_{1}\left(\rho_{0}-\rho_{1}\right)} & \frac{\lambda\left(\rho_{0}^{k+1}-\rho_{1}^{k+1}\right)}{\beta_{1}\left(\rho_{0}-\rho_{1}\right)} \\
\frac{\lambda\left(\lambda+\beta_{2}\right)\left(\rho_{0}^{k+1}-\rho_{1}^{k+1}\right)}{\mu \beta_{1}\left(\rho_{0}-\rho_{1}\right)} & \frac{\lambda}{\beta_{1}}\left(\frac{\mu \beta_{1}\left(\rho_{0}^{k+1}-\rho_{1}^{k+1}\right)-\lambda \beta_{1}\left(\rho_{0}^{k}-\rho_{1}^{k}\right)}{\mu \beta_{1}\left(\rho_{0}-\rho_{1}\right)}\right)
\end{array}\right] \\
& =\left[\begin{array}{cc}
\frac{\lambda\left(\rho_{0}^{k+1}-\rho_{1}^{k+1}\right)}{\beta_{1}\left(\rho_{0}-\rho_{1}\right)} \\
\frac{\lambda\left(\lambda+\beta_{2}\right)\left(\rho_{0}^{k+1}-\rho_{1}^{k+1}\right)}{\mu \beta_{1}\left(\rho_{0}-\rho_{1}\right)} & \frac{\lambda\left(\rho_{0}^{k+1}-\rho_{1}^{k+1}\right)-\beta_{1} \rho_{0} \rho_{1}\left(\rho_{0}^{k}-\rho_{1}^{k}\right)}{\beta_{1}\left(\rho_{0}-\rho_{1}\right)}
\end{array}\right] \\
& =\left[\begin{array}{cc}
\frac{\left(\beta_{1} \rho_{0}-\lambda\right) \rho_{0}^{k+1}+\rho_{1}^{k+1}\left(\lambda-\beta_{1} \rho_{1}\right)}{\beta_{1}\left(\rho_{0}-\rho_{1}\right)} & \frac{\lambda\left(\rho_{0}^{k+1}-\rho_{1}^{k+1}\right)}{\beta_{1}\left(\rho_{0}-\rho_{1}\right)} \\
\frac{\lambda\left(\lambda+\beta_{2}\right)\left(\rho_{0}^{k+1}-\rho_{1}^{k+1}\right)}{\mu \beta_{1}\left(\rho_{0}-\rho_{1}\right)} & \frac{\left(\lambda-\beta_{1} \rho_{1}\right) \rho_{0}^{k+1}+\rho_{1}^{k+1}\left(\beta_{1} \rho_{0}-\lambda\right)}{\beta_{1}\left(\rho_{0}-\rho_{1}\right)}
\end{array}\right]=\mathbf{R}^{k+1}
\end{aligned}
$$

Remark. Two substitutions were needed in this derivation, namely: $\rho_{0}+\rho_{1}=\frac{\lambda\left(\lambda+\mu+\beta_{1}+\beta_{2}\right)}{\mu \beta_{1}}$ and $\rho_{0} \rho_{1}=\frac{\lambda^{2}}{\mu \beta_{1}}$. These can easily be verified from (9).

\subsection{The Initial Terms of $\pi$}

Next we turn our attention to computing $\mathbf{B}[\mathbf{R}]$, and a positive vector $\left(x_{0}, \mathbf{x}_{1}\right)$, such that $\left(x_{0}, \mathbf{x}_{1}\right) \mathbf{B}[\mathbf{R}]=0$ :

$$
\begin{aligned}
\left(x_{0}, \mathbf{x}_{1}\right) \mathbf{B}[\mathbf{R}]=\left(x_{0}, \mathbf{x}_{\mathbf{1}}\right)\left[\begin{array}{cc}
\hat{\mathbf{A}} & \hat{\mathbf{C}} \\
\hat{\mathbf{B}} & \mathbf{A}+\mathbf{R B}
\end{array}\right]=\left(x_{0}, \mathbf{x}_{1}\right)\left[\begin{array}{ccc}
-\lambda & \lambda & 0 \\
0 & -\mu & \mu \\
\beta_{1} & \lambda+\beta_{2} & -\left(\lambda+\beta_{1}+\beta_{2}\right)
\end{array}\right]=\mathbf{0} \\
\Longrightarrow\left(x_{0}, \mathbf{x}_{\mathbf{1}}\right)=\left(1,\left(\frac{\lambda\left(\beta_{1}+\beta_{2}+\lambda\right)}{\beta_{1} \mu}, \frac{\lambda}{\beta_{1}}\right)\right)
\end{aligned}
$$

We now seek to normalize the solution in order to generate the first three terms of $\pi$ :

$$
\begin{gathered}
\mathrm{K}\left(\mathbf{x}_{\mathbf{0}} \mathbf{e}+\mathbf{x}_{\mathbf{1}}(\mathbf{I}-\mathbf{R})^{-1} \mathbf{e}\right)=1 \Longrightarrow \mathrm{K}=\frac{\beta_{1}(\mu-\lambda)-\lambda\left(\beta_{2}+\mu\right)}{\beta_{1} \mu}=\left(1-\rho_{0}\right)\left(1-\rho_{1}\right) \\
\Longrightarrow\left(\pi_{00}, \pi_{10}, \pi_{11}\right)=\mathrm{K}\left(\mathrm{x}_{0}, \mathbf{x}_{\mathbf{1}}\right) \Longrightarrow\left\{\begin{array}{l}
\pi_{00}=\frac{\beta_{1}(\mu-\lambda)-\lambda\left(\beta_{2}+\mu\right)}{\beta_{1} \mu}=\mathrm{K} \\
\pi_{10}=\frac{\lambda\left(\beta_{1}+\beta_{2}+\lambda\right)\left(\beta_{1}(\mu-\lambda)-\lambda\left(\beta_{2}+\mu\right)\right)}{\beta_{1}^{2} \mu^{2}}=\frac{\lambda K\left(\beta_{1}+\beta_{2}+\lambda\right)}{\beta_{1} \mu} \\
\pi_{11}=\frac{\lambda\left(\beta_{1}(\mu-\lambda)-\lambda\left(\beta_{2}+\mu\right)\right)}{\beta_{1}^{2} \mu}=\frac{\lambda K}{\beta_{1}}
\end{array}\right.
\end{gathered}
$$

Remark. We observe that the condition given by the Corollary to Lemma 4.1 for positive recurrence: $\beta_{1}(\mu-\lambda)-\lambda(\mu+$ $\left.\beta_{2}\right)>0$ is equivalent to $K>0$.

Proposition 7.2. The remaining elements $\left\{\left(\pi_{\mathrm{k} 0}, \pi_{\mathrm{k} 1}\right) \mid \mathrm{k} \geqslant 2\right\}$ of our stationary distribution satisfying $\left(\pi_{\mathrm{k} 0}, \pi_{\mathrm{k} 1}\right)=$ $\left(\pi_{10}, \pi_{11}\right) \mathbf{R}^{\mathrm{k}-1}$ and $\pi_{00}+\sum_{\mathrm{k}=1}^{\infty}\left(\pi_{\mathrm{k} 0}+\pi_{\mathrm{k} 1}\right)=1$ are given by:

$$
\left\{\begin{array}{l}
\pi_{\mathrm{k} 0}=\mathrm{K}\left(\frac{\rho_{0}^{\mathrm{k}+1}-\rho_{1}^{\mathrm{k}+1}}{\rho_{0}-\rho_{1}}-\frac{\lambda\left(\rho_{0}^{\mathrm{k}}-\rho_{1}^{\mathrm{k}}\right)}{\beta_{1}\left(\rho_{0}-\rho_{1}\right)}\right) \\
\pi_{\mathrm{k} 1}=\frac{\lambda K\left(\rho_{0}^{\mathrm{k}}-\rho_{1}^{\mathrm{k}}\right)}{\beta_{1}\left(\rho_{0}-\rho_{1}\right)}
\end{array}\right.
$$

Proof. To motivate the proof, we begin by noting that:

$$
\begin{aligned}
\left(\pi_{\mathrm{k} 0}, \pi_{\mathrm{k} 1}\right)=\left(\pi_{10}, \pi_{11}\right) \mathbf{R}^{\mathrm{k}-1} & \Longleftrightarrow\left(\pi_{\mathrm{k} 0}, \pi_{\mathrm{k} 1}\right)=\left(\pi_{10}, \pi_{11}\right) \mathbf{R}^{\mathrm{k}-2} \mathbf{R} \\
& \Longleftrightarrow\left(\pi_{\mathrm{k} 0}, \pi_{\mathrm{k} 1}\right)=\left(\pi_{\mathrm{k}-1,0}, \pi_{\mathrm{k}-1,1}\right) \mathbf{R}
\end{aligned}
$$

and consider:

$$
\begin{aligned}
& \left(\pi_{k-1,0}, \pi_{k-1,1}\right) \mathbf{R}=\frac{\lambda K}{\beta_{1}}\left(\frac{\rho_{0}^{k}-\rho_{1}^{k}}{\rho_{0}-\rho_{1}}-\frac{\lambda\left(\rho_{0}^{k-1}-\rho_{1}^{k-1}\right)}{\beta_{1}\left(\rho_{0}-\rho_{1}\right)}, \frac{\lambda\left(\rho_{0}^{k-1}-\rho_{1}^{k-1}\right)}{\beta_{1}\left(\rho_{0}-\rho_{1}\right)}\right)\left[\begin{array}{cc}
\frac{\lambda+\beta_{1}+\beta_{2}}{\mu} & 1 \\
\frac{\lambda+\beta_{2}}{\mu} & 1
\end{array}\right] \\
& =\frac{\lambda K}{\beta_{1}}\left(\frac{\lambda+\beta_{1}+\beta_{2}}{\mu} \frac{\rho_{0}^{k}-\rho_{1}^{k}}{\rho_{0}-\rho_{1}}-\frac{\lambda+\beta_{1}+\beta_{2}}{\mu} \frac{\lambda\left(\rho_{0}^{k-1}-\rho_{1}^{k-1}\right)}{\beta_{1}\left(\rho_{0}-\rho_{1}\right)}+\frac{\lambda+\beta_{2}}{\mu} \frac{\lambda\left(\rho_{0}^{k-1}-\rho_{1}^{k-1}\right)}{\beta_{1}\left(\rho_{0}-\rho_{1}\right)},\right. \\
& \left.\frac{\rho_{0}^{k}-\rho_{1}^{k}}{\rho_{0}-\rho_{1}}-\frac{\lambda\left(\rho_{0}^{k-1}-\rho_{1}^{k-1}\right)}{\beta_{1}\left(\rho_{0}-\rho_{1}\right)}+\frac{\lambda\left(\rho_{0}^{k-1}-\rho_{1}^{k-1}\right)}{\beta_{1}\left(\rho_{0}-\rho_{1}\right)}\right) \\
& =\frac{\lambda K}{\beta_{1}}\left(\frac{\lambda+\beta_{1}+\beta_{2}}{\mu} \frac{\rho_{0}^{k}-\rho_{1}^{k}}{\rho_{0}-\rho_{1}}-\frac{\beta_{1}}{\mu} \frac{\lambda\left(\rho_{0}^{k-1}-\rho_{1}^{k-1}\right)}{\beta_{1}\left(\rho_{0}-\rho_{1}\right)}, \frac{\rho_{0}^{k}-\rho_{1}^{k}}{\rho_{0}-\rho_{1}}\right)
\end{aligned}
$$


We have:

$$
\begin{aligned}
& =K\left(\frac{\lambda\left(\lambda+\beta_{1}+\beta_{2}\right)}{\mu \beta_{1}} \frac{\rho_{0}^{k}-\rho_{1}^{k}}{\rho_{0}-\rho_{1}}-\frac{\lambda^{2}\left(\rho_{0}^{k-1}-\rho_{1}^{k-1}\right)}{\mu \beta_{1}\left(\rho_{0}-\rho_{1}\right)}, \frac{\lambda\left(\rho_{0}^{k}-\rho_{1}^{k}\right)}{\beta_{1}\left(\rho_{0}-\rho_{1}\right)}\right) \\
& =K\left(\frac{\lambda\left(\lambda+\mu+\beta_{1}+\beta_{2}\right)}{\mu \beta_{1}} \frac{\rho_{0}^{k}-\rho_{1}^{k}}{\rho_{0}-\rho_{1}}-\frac{\lambda\left(\rho_{0}^{k}-\rho_{1}^{k}\right)}{\beta_{1}\left(\rho_{0}-\rho_{1}\right)}-\frac{\lambda^{2}\left(\rho_{0}^{k-1}-\rho_{1}^{k-1}\right)}{\mu \beta_{1}\left(\rho_{0}-\rho_{1}\right)}, \frac{\lambda\left(\rho_{0}^{k}-\rho_{1}^{k}\right)}{\beta_{1}\left(\rho_{0}-\rho_{1}\right)}\right) \\
& =K\left(\frac{\left(\rho_{0}+\rho_{1}\right)\left(\rho_{0}^{k}-\rho_{1}^{k}\right)}{\rho_{0}-\rho_{1}}-\frac{\lambda\left(\rho_{0}^{k}-\rho_{1}^{k}\right)}{\beta_{1}\left(\rho_{0}-\rho_{1}\right)}-\frac{\rho_{0} \rho_{1}\left(\rho_{0}^{k-1}-\rho_{1}^{k-1}\right)}{\left(\rho_{0}-\rho_{1}\right)}, \frac{\lambda\left(\rho_{0}^{k}-\rho_{1}^{k}\right)}{\beta_{1}\left(\rho_{0}-\rho_{1}\right)}\right) \\
& =K\left(\frac{\rho_{0}^{k+1}-\rho_{1}^{k+1}}{\rho_{0}-\rho_{1}}-\frac{\lambda\left(\rho_{0}^{k}-\rho_{1}^{k}\right)}{\beta_{1}\left(\rho_{0}-\rho_{1}\right)}, \frac{\lambda\left(\rho_{0}^{k}-\rho_{1}^{k}\right)}{\beta_{1}\left(\rho_{0}-\rho_{1}\right)}\right)=\left(\pi_{k 0}, \pi_{k 1}\right)
\end{aligned}
$$

$\pi_{00}+\sum_{k=1}^{\infty}\left(\pi_{k 0}+\pi_{k 1}\right)=\sum_{k=0}^{\infty}\left(\pi_{k 0}+\pi_{k 1}\right)$, where $(13)$ is valid for all $k \in \mathbb{N} \cup\{0\}$

$$
\begin{aligned}
& =\mathrm{K} \sum_{k=0}^{\infty}\left(\frac{\rho_{0}^{k+1}-\rho_{1}^{k+1}}{\rho_{0}-\rho_{1}}-\frac{\lambda\left(\rho_{0}^{k}-\rho_{1}^{k}\right)}{\beta_{1}\left(\rho_{0}-\rho_{1}\right)}+\frac{\lambda\left(\rho_{0}^{k}-\rho_{1}^{k}\right)}{\beta_{1}\left(\rho_{0}-\rho_{1}\right)}\right) \\
& =K \sum_{k=0}^{\infty}\left(\frac{\rho_{0}^{k+1}-\rho_{1}^{k+1}}{\rho_{0}-\rho_{1}}\right) \\
& =\frac{K}{\rho_{0}-\rho_{1}} \sum_{k=0}^{\infty} \rho_{0}^{k+1}-\sum_{k=0}^{\infty} \rho_{1}^{k+1} \\
& =\frac{K}{\rho_{0}-\rho_{1}}\left(\frac{\rho_{0}}{1-\rho_{0}}-\frac{\rho_{1}}{1-\rho_{1}}\right) \\
& =\frac{K}{\rho_{0}-\rho_{1}}\left(\frac{\rho_{0}\left(1-\rho_{1}\right)-\rho_{1}\left(1-\rho_{0}\right)}{\left(1-\rho_{0}\right)\left(1-\rho_{1}\right)}\right) \\
& =\frac{K}{\rho_{0}-\rho_{1}}\left(\frac{\rho_{0}-\rho_{1}}{\left(1-\rho_{0}\right)\left(1-\rho_{1}\right)}\right) \\
& =\frac{K}{\left(1-\rho_{0}\right)\left(1-\rho_{1}\right)}=1, \quad \text { since } K=\left(1-\rho_{0}\right)\left(1-\rho_{1}\right) .
\end{aligned}
$$

\section{Decomposition}

\subsection{Decomposition of $N$}

Theorem 8.1. If $\mathrm{K}>0$, then the stationary number of customers in steady-state, $\mathrm{N}$, can be decomposed into the sum of two independent geometric random variables parameterized by $1-\rho_{0}$ and $1-\rho_{1}$. Namely: $\mathrm{N}=\mathrm{X}_{0}+\mathrm{X}_{1}$, where:

$\mathrm{X}_{0} \sim \operatorname{Geometric}\left(1-\rho_{0}\right)$, and $\mathrm{X}_{1} \sim \operatorname{Geometric}\left(1-\rho_{1}\right)$.

Proof. With our stationary distribution explicitly found, we compute the stationary queue-length probability generating function (P.G.F.) defined by:

$$
\begin{aligned}
\mathcal{G}_{\mathcal{N}}(z)=\sum_{k=0}^{\infty} \mathrm{P}(\mathrm{N}=\mathrm{k}) z^{\mathrm{k}} \\
\Longrightarrow \mathcal{G}_{\mathcal{N}}(z)=\sum_{\mathrm{k}=0}^{\infty} \mathrm{P}(\mathrm{N}=\mathrm{k}) z^{\mathrm{k}}=\sum_{\mathrm{k}=0}^{\infty} \mathrm{P}((\mathrm{N}=\mathrm{k} \cap \mathrm{S}=0) \cup(\mathrm{N}=\mathrm{k} \cap \mathrm{S}=1)) z^{\mathrm{k}} \\
=\sum_{\mathrm{k}=0}^{\infty}(\mathrm{P}(\mathrm{N}=\mathrm{k} \cap \mathrm{S}=0)+\mathrm{P}(\mathrm{N}=\mathrm{k} \cap \mathrm{S}=1)) z^{\mathrm{k}} \\
=\sum_{\mathrm{k}=0}^{\infty}\left(\pi_{\mathrm{k} 0}+\pi_{\mathrm{k} 1}\right) z^{\mathrm{k}} \\
=\mathrm{K} \sum_{\mathrm{k}=0}^{\infty}\left(\frac{\rho_{0}^{\mathrm{k}+1}-\rho_{1}^{k+1}}{\rho_{0}-\rho_{1}}-\frac{\lambda\left(\rho_{0}^{\mathrm{k}}-\rho_{1}^{\mathrm{k}}\right)}{\beta_{1}\left(\rho_{0}-\rho_{1}\right)}+\frac{\lambda\left(\rho_{0}^{\mathrm{k}}-\rho_{1}^{\mathrm{k}}\right)}{\beta_{1}\left(\rho_{0}-\rho_{1}\right)}\right) z^{\mathrm{k}} \\
=\mathrm{K} \sum_{\mathrm{k}=0}^{\infty}\left(\frac{\rho_{0}^{\mathrm{k}+1}-\rho_{1}^{k+1}}{\rho_{0}-\rho_{1}}\right) z^{\mathrm{k}} \\
=\frac{\mathrm{K}}{\rho_{0}-\rho_{1}} \sum_{\mathrm{k}=0}^{\infty}\left(\rho_{0}^{\mathrm{k}+1}-\rho_{1}^{\mathrm{k}+1}\right) z^{\mathrm{k}} \\
=\frac{\mathrm{K}}{\rho_{0}-\rho_{1}}\left(\rho_{0} \sum_{\mathrm{k}=0}^{\infty}\left(\rho_{0} z\right)^{\mathrm{k}}-\rho_{1} \sum_{\mathrm{k}=0}^{\infty}\left(\rho_{1} z\right)^{\mathrm{k}}\right) \\
=\frac{\mathrm{K}}{\rho_{0}-\rho_{1}}\left(\frac{\rho_{0}}{1-\rho_{0} z}-\frac{\rho_{1}}{1-\rho_{1} z}\right) \\
=\frac{\mathrm{K}}{\rho_{0}-\rho_{1}}\left(\frac{\rho_{0}\left(1-\rho_{1} z\right)-\rho_{1}\left(1-\rho_{0} z\right)}{\left(1-\rho_{0} z\right)\left(1-\rho_{1} z\right)}\right)
\end{aligned}
$$




$$
\begin{aligned}
= & \frac{\mathrm{K}}{\rho_{0}-\rho_{1}}\left(\frac{\rho_{0}-\rho_{1}}{\left(1-\rho_{0} z\right)\left(1-\rho_{1} z\right)}\right) \\
= & \frac{\mathrm{K}}{\left(1-\rho_{0} z\right)\left(1-\rho_{1} z\right)} \\
= & \frac{1-\rho_{0}}{1-\rho_{0} z} \frac{1-\rho_{1}}{1-\rho_{1} z}=\mathcal{G X}_{0}(z) \mathcal{G X}_{1}(z) \\
\Longrightarrow & N=X_{0}+X_{1}, \text { where } X_{0} \text { and } X_{1} \text { are independent, } \\
& \quad X_{0} \sim \operatorname{Geometric}\left(1-\rho_{0}\right), \text { and } \\
& \quad X_{1} \sim \operatorname{Geometric}\left(1-\rho_{1}\right) .
\end{aligned}
$$

\subsection{Little's Distributional Law}

Since we have the P.G.F. of N, we may now employ Little's Distributional Law, named after John Little for his work in 1961 (Little, 1961), proved in general by Keilson, J. \& Servi, L.D. in 1988 (Keilson \& Servi, 1988).

\section{Theorem 8.2. Little's Distributional Law}

Let $\mathrm{N}$ be the stationary number of customers in a steady-state queue where the arrivals come according to a Poisson stream with rate $\lambda$. Let $\mathrm{W}$ be the stationary waiting time. Let $\mathcal{W}^{*}(\mathrm{~s})$ denote the L.S.T. (Laplace Stieltjes Transform) of W. Then:

$$
\mathcal{G}_{\mathrm{N}}(z)=\mathcal{W}^{*}((1-z) \lambda)
$$

Proof. While we will refer the reader to Keilson, J. \& Servi, L.D. (Keilson \& Servi, 1988) for details, we give an elementary direct proof in line with our notation. Given the definition of the P.G.F. of $N$, we rewrite $P(N=k)$ via total probability and obtain:

$$
\begin{aligned}
\mathcal{G}_{N}(z) & =\sum_{k=0}^{\infty} \mathrm{P}(\mathrm{N}=\mathrm{k}) z^{k}=\sum_{k=0}^{\infty} z^{k} \int_{-\infty}^{\infty} \mathrm{P}(\mathrm{N}=\mathrm{k} \mid \mathrm{W}=\mathrm{t}) d \mathrm{~F}_{W}=\sum_{k=0}^{\infty} z^{k} \int_{-\infty}^{\infty} e^{-\lambda t} \frac{(\lambda t)^{k}}{k !} d F_{W} \\
& =\int_{-\infty}^{\infty} e^{-\lambda t} \sum_{k=0}^{\infty} \frac{(\lambda t z)^{k}}{k !} d F_{W}=\int_{-\infty}^{\infty} e^{-\lambda t} e^{\lambda t z} d F_{W}=\int_{-\infty}^{\infty} e^{-\lambda t(1-z)} d F_{W}=\mathcal{W}^{*}((1-z) \lambda)
\end{aligned}
$$

\subsection{Decomposition of $W$}

Theorem 8.3. If $\mathrm{K}>0$, then the stationary waiting time $\mathrm{W}$ for customers in the steady-state queue of length $\mathrm{N}$ can be decomposed into the sum of two independent exponential random variables parameterized by $\frac{\lambda\left(1-\rho_{0}\right)}{\rho_{0}}$ and $\frac{\lambda\left(1-\rho_{1}\right)}{\rho_{1}}$. Namely: $\mathrm{W}=\mathrm{Y}_{0}+\mathrm{Y}_{1}$, where: $\mathrm{Y}_{0} \sim \operatorname{Exponential}\left(\frac{\lambda\left(1-\rho_{0}\right)}{\rho_{0}}\right)$, and $\mathrm{Y}_{1} \sim \operatorname{Exponential}\left(\frac{\lambda\left(1-\rho_{1}\right)}{\rho_{1}}\right)$.

Proof. Using Theorem 8.2, we can find the L.S.T. of W explicitly:

$$
\begin{aligned}
\mathcal{W}^{*}(\mathrm{~s})=\mathcal{G}_{N}\left(1-\frac{\mathrm{s}}{\lambda}\right) & =\frac{K}{\left(1-\rho_{0}\left(1-\frac{s}{\lambda}\right)\right)\left(1-\rho_{1}\left(1-\frac{s}{\lambda}\right)\right)} \\
& =\frac{K \lambda^{2}}{\left(\lambda-\rho_{0}(\lambda-s)\right)\left(\lambda-\rho_{1}(\lambda-s)\right)} \\
& =\frac{K \lambda^{2}}{\left(\lambda\left(1-\rho_{0}\right)+\rho_{0} s\right)\left(\lambda\left(1-\rho_{1}\right)+\rho_{1} s\right)} \\
& =\frac{\frac{K \lambda^{2}}{\rho_{0} \rho_{1}}}{\left(\frac{\lambda\left(1-\rho_{0}\right)}{\rho_{0}}+s\right)\left(\frac{\lambda\left(1-\rho_{1}\right)}{\rho_{1}}+s\right)} \\
& =\frac{\frac{\lambda\left(1-\rho_{0}\right)}{\rho_{0}} \frac{\lambda\left(1-\rho_{1}\right)}{\rho_{1}}}{\left(\frac{\lambda\left(1-\rho_{0}\right)}{\rho_{0}}+s\right)\left(\frac{\lambda\left(1-\rho_{1}\right)}{\rho_{1}}+s\right)} \\
& =\frac{\frac{\lambda\left(1-\rho_{0}\right)}{\rho_{0}}}{\left(\frac{\lambda\left(1-\rho_{0}\right)}{\rho_{0}}+s\right)} \frac{\frac{\lambda\left(1-\rho_{1}\right)}{\rho_{1}}}{\left(\frac{\lambda\left(1-\rho_{1}\right)}{\rho_{1}}+s\right)} \\
& \Longrightarrow W^{=}=Y_{0}+Y_{1}, \text { where } Y_{0} \text { and } Y_{1} \text { are independent, } \\
& Y_{0} \sim \text { Exponential }\left(\frac{\lambda\left(1-\rho_{0}\right)}{\rho_{0}}\right), \text { and } Y_{1} \sim \text { Exponential }\left(\frac{\lambda\left(1-\rho_{1}\right)}{\rho_{1}}\right) .
\end{aligned}
$$




\section{Results}

Table 1. Analytical Results

\begin{tabular}{lrr}
\hline & $\begin{array}{r}\text { Steady-State \# of Customers: } N \\
\text { Steady-State Waiting Time: } W\end{array}$ \\
& $\mathcal{G}_{N}(z)=\frac{1-\rho_{0}}{1-\rho_{0} z} \frac{1-\rho_{1}}{1-\rho_{1} z}$ & $\mathcal{W}^{*}(s)=\frac{\frac{\lambda\left(1-\rho_{0}\right)}{\rho_{0}}}{\left(\frac{\lambda\left(-\rho_{0}\right)}{\rho_{0}}+s\right)} \frac{\frac{\lambda\left(1-\rho_{1}\right)}{\rho_{1}}}{\left(\frac{\lambda\left(1-\rho_{1}\right)}{\rho_{1}}+s\right)}$ \\
\hline $\mathrm{E}()$. & $\frac{\rho_{0}}{1-\rho_{0}}+\frac{\rho_{1}}{1-\rho_{1}}$ & $\frac{\rho_{0}}{\lambda\left(1-\rho_{0}\right)}+\frac{\rho_{1}}{\lambda\left(1-\rho_{1}\right)}$ \\
$\operatorname{Var}()$. & $\frac{\rho_{0}}{\left(1-\rho_{0}\right)^{2}}+\frac{\rho_{1}}{\left(1-\rho_{1}\right)^{2}}$ & $\frac{\rho_{0}^{2}}{\lambda^{2}\left(1-\rho_{0}\right)^{2}}+\frac{\rho_{1}^{2}}{\lambda^{2}\left(1-\rho_{1}\right)^{2}}$ \\
\hline
\end{tabular}

\section{Special cases}

We can now recover the stationary behavior of the following known queue types as particular cases from our model as follows:

- $\beta_{1} \rightarrow \infty$ with $0 \leqslant \beta_{2}<\infty$ results in the classical $M / M / 1$ queue.

- $0<\beta_{1}<\infty$ with $\beta_{2}=0$ and $\mu=\beta_{1}$ results in an $M / E_{2} / 1$ queue, where $E_{2}$ refers to an 'Erlang' service time distribution with shape 2 and rate $\mu$.

- $0<\beta_{1}<\infty$ with $\beta_{2}=0$ and $\mu>\beta_{1}$ results in an $M / H E / 1$ queue, where HE refers to a hypoexponential service time distribution $\sim f(t)=\frac{\mu \beta_{1}\left(e^{-\beta_{1} t}-e^{-\mu t}\right)}{\mu-\beta_{1}}$ (pg. 308 in Ross, 2006).

$10.1 \mathrm{M} / \mathrm{M} / 1$

\section{Proposition 10.1.}

If $0 \leqslant \beta_{2}<\infty$ and $\beta_{1} \rightarrow \infty$, our model recovers the stationary behavior of the classical $M / M / 1$.

Proof.

We begin by computing the eigenvalues of $\mathbf{R}$ under these conditions.

$\lim _{\beta_{1} \rightarrow \infty} \rho_{0}=\lim _{\beta_{1} \rightarrow \infty} \frac{\lambda\left(\lambda+\mu+\beta_{1}+\beta_{2}+\sqrt{\left(\lambda+\mu+\beta_{1}+\beta_{2}\right)^{2}-4 \mu \beta_{1}}\right)}{2 \mu \beta_{1}}=\frac{\lambda}{\mu}=\rho<1 \Longrightarrow$ Positive Recurrent

$\lim _{\beta_{1} \rightarrow \infty} \rho_{1}=\lim _{\beta_{1} \rightarrow \infty} \frac{\lambda\left(\lambda+\mu+\beta_{1}+\beta_{2}-\sqrt{\left(\lambda+\mu+\beta_{1}+\beta_{2}\right)^{2}-4 \mu \beta_{1}}\right)}{2 \mu \beta_{1}}=0$

We now consider our P.G.F. with the appropriate substitutions.

$\mathcal{G}_{\mathrm{N}}(z)=\frac{1-\rho_{0}}{1-\rho_{0} z} \frac{1-\rho_{1}}{1-\rho_{1} z}=\frac{1-\rho}{1-\rho z}$

Since the P.G.F. of $\mathrm{N}$ matches that of the classical M/M/1 queue given on page 32 of Adan, I., \& Resing, J. (Adan \& Resing, 2001), we conclude that the stationary queue lengths are equivalent in distribution.

$10.2 \mathrm{M} / \mathrm{E}_{2} / \mathrm{l}$

\section{Proposition 10.2.}

If $0<\beta_{1}<\infty, \beta_{2}=0$ and $\mu=\beta_{1}$, our model recovers the stationary behavior of an $M / E_{2} / 1$ queue, where $E_{2}$ refers to an 'Erlang' service time distribution with shape 2 and rate $\mu$.

Proof.

We begin, again, by computing the eigenvalues of $\mathbf{R}$ under these conditions and obtain:

$\rho_{0}=\frac{\lambda\left(\lambda+\mu+\beta_{1}+\beta_{2}+\sqrt{\left(\lambda+\mu+\beta_{1}+\beta_{2}\right)^{2}-4 \mu \beta_{1}}\right)}{2 \mu \beta_{1}}=\frac{\lambda\left(\lambda+2 \mu+\sqrt{(\lambda+2 \mu)^{2}-4 \mu^{2}}\right)}{2 \mu^{2}}=\frac{\lambda(\lambda+2 \mu+\sqrt{\lambda(\lambda+4 \mu)})}{2 \mu^{2}}$

We also note that $\frac{\lambda(\lambda+2 \mu+\sqrt{\lambda(\lambda+4 \mu)})}{2 \mu^{2}}<1 \Longleftrightarrow \frac{2 \lambda}{\mu}<1$

Therefore, let $\rho=\frac{2 \lambda}{\mu}$ and we have $\rho_{0}<1 \Longleftrightarrow \rho<1 \Longrightarrow$ Positive Recurrent

We must now consider our L.S.T. $\mathcal{W}^{*}(s)$ :

$$
\begin{aligned}
\mathcal{W}^{*}(\mathrm{~s})=\mathcal{G}_{\mathrm{N}}\left(1-\frac{\mathrm{s}}{\lambda}\right) & =\frac{\mathrm{K}}{\left(1-\rho_{0}\left(1-\frac{s}{\lambda}\right)\right)\left(1-\rho_{1}\left(1-\frac{\mathrm{s}}{\lambda}\right)\right)} \\
& =\frac{\mathrm{K} \lambda^{2}}{\left(\lambda-\rho_{0}(\lambda-s)\right)\left(\lambda-\rho_{1}(\lambda-s)\right)} \\
& =\frac{\mathrm{K} \lambda^{2}}{\left(\lambda\left(1-\rho_{0}\right)+\rho_{0} s\right)\left(\lambda\left(1-\rho_{1}\right)+\rho_{1} s\right)}
\end{aligned}
$$




$$
\begin{aligned}
& =\frac{K \lambda^{2}}{\lambda^{2}\left(1-\rho_{0}\right)\left(1-\rho_{1}\right)+\lambda \rho_{1}\left(1-\rho_{0}\right) s+\lambda \rho_{0}\left(1-\rho_{1}\right) s+\rho_{0} \rho_{1} s^{2}} \\
& =\frac{K \lambda^{2}}{\lambda^{2}\left(1-\rho_{0}\right)\left(1-\rho_{1}\right)+\lambda \rho_{1} s-\lambda \rho_{0} \rho_{1} s+\lambda \rho_{0} s-\lambda \rho_{0} \rho_{1} s+\rho_{0} \rho_{1} s^{2}} \\
& =\frac{K \lambda^{2}}{\lambda^{2}\left(1-\rho_{0}\right)\left(1-\rho_{1}\right)+\lambda s\left(\rho_{0}+\rho_{1}\right)+\rho_{0} \rho_{1}\left(s^{2}-2 \lambda s\right)}
\end{aligned}
$$

We now need some substitutions: namely:

$K=\left(1-\rho_{0}\right)\left(1-\rho_{1}\right)=\frac{\beta_{1}(\mu-\lambda)-\lambda\left(\beta_{2}+\mu\right)}{\beta_{1} \mu}=\frac{\mu(\mu-\lambda)-\lambda(\mu)}{\mu^{2}}=\frac{\mu^{2}-2 \lambda \mu}{\mu^{2}}$

$\rho_{0} \rho_{1}=\frac{\lambda^{2}}{\mu \beta_{1}}=\frac{\lambda^{2}}{\mu^{2}}$

$\rho_{0}+\rho_{1}=\frac{\lambda\left(\lambda+\mu+\beta_{1}+\beta_{2}+\sqrt{\left(\lambda+\mu+\beta_{1}+\beta_{2}\right)^{2}-4 \mu \beta_{1}}\right)}{2 \mu \beta_{1}}+\frac{\lambda\left(\lambda+\mu+\beta_{1}+\beta_{2}-\sqrt{\left(\lambda+\mu+\beta_{1}+\beta_{2}\right)^{2}-4 \mu \beta_{1}}\right)}{2 \mu \beta_{1}}=\frac{2 \lambda\left(\lambda+\mu+\beta_{1}+\beta_{2}\right)}{2 \mu \beta_{1}}=\frac{\lambda^{2}+2 \lambda \mu}{\mu^{2}}$

Proceeding where we left off, we have:

$$
\begin{aligned}
\mathcal{W}^{*}(s)=\mathcal{G}_{N}\left(1-\frac{s}{\lambda}\right) & =\frac{\frac{\lambda^{2} \mu^{2}-2 \lambda^{3} \mu}{\mu^{2}}}{\lambda^{2} \frac{\mu^{2}-2 \lambda \mu}{\mu^{2}}+\lambda s \frac{\lambda^{2}+2 \lambda \mu}{\mu^{2}}+\frac{\lambda^{2}}{\mu^{2}}\left(s^{2}-2 \lambda s\right)}=\frac{\mu^{2}-2 \lambda \mu}{\mu^{2}-2 \lambda \mu+s(\lambda+2 \mu)+\left(s^{2}-2 \lambda s\right)}=\frac{\mu^{2}-2 \lambda \mu}{\mu^{2}-2 \lambda \mu+\lambda s+2 \mu s+s^{2}-2 \lambda s} \\
& =\frac{\mu^{2} s(1-\rho)}{\mu^{2} s-2 \lambda \mu s+2 \mu s^{2}+s^{3}-\lambda s^{2}} \\
& =\frac{\mu^{2} s(1-\rho)}{-\lambda s^{2}-2 \lambda \mu s-\lambda \mu^{2}+\lambda \mu^{2}+2 \mu s^{2}+s^{3}+\mu^{2} s} \\
& =\frac{\mu^{2} s(1-\rho)}{-\lambda(s+\mu)^{2}+s^{3}+2 \mu s^{2}+\mu^{2} s+\lambda \mu^{2}} \\
& =\frac{\mu^{2} s(1-\rho)}{-\lambda(s+\mu)^{2}+s(s+\mu)^{2}+\lambda \mu^{2}} \\
& =\frac{s(1-\rho)\left(\frac{\mu}{s+\mu}\right)^{2}}{s-\lambda+\lambda\left(\frac{\mu}{s+\mu}\right)^{2}} \text { which matches what is given on page } 85 \text { of Ibe, Oliver (Ibe, 2011). }
\end{aligned}
$$

Therefore, we conclude that the stationary queue length is equivalent in distribution to an $M / E_{2} / 1$.

\section{$10.3 \mathrm{M} / \mathrm{HE} / 1$}

\section{Proposition 10.3.}

If $0<\beta_{1}<\infty, \beta_{2}=0$ and $\mu>\beta_{1}$ we have the stationary behavior of an $\mathrm{M} / \mathrm{HE} / 1$ queue, where $\mathrm{HE}$ refers to a hypoexponential service time $\sim \mathrm{f}(\mathrm{t})=\frac{\mu \beta_{1}\left(\mathrm{e}^{-\beta_{1} \mathrm{t}}-\mathrm{e}^{-\mu \mathrm{t}}\right)}{\mu-\beta_{1}}$ (see pg. 308 in Ross, 2006).

\section{Proof.}

We once more compute the eigenvalues of $\mathbf{R}$ under these given conditions and obtain:

$\rho_{0}=\frac{\lambda\left(\lambda+\mu+\beta_{1}+\beta_{2}+\sqrt{\left(\lambda+\mu+\beta_{1}+\beta_{2}\right)^{2}-4 \mu \beta_{1}}\right)}{2 \mu \beta_{1}}=\frac{\lambda\left(\lambda+\mu+\beta_{1}+\sqrt{\left(\lambda+\mu+\beta_{1}\right)^{2}-4 \mu \beta_{1}}\right)}{2 \mu \beta_{1}}$

We again note that $\frac{\lambda\left(\lambda+\mu+\beta_{1}+\sqrt{\left(\lambda+\mu+\beta_{1}\right)^{2}-4 \mu \beta_{1}}\right)}{2 \mu \beta_{1}}<1 \Longleftrightarrow \frac{\lambda\left(\mu+\beta_{1}\right)}{\mu \beta_{1}}<1$

Therefore, for notational convenience, we will let $\rho=\frac{\lambda\left(\mu+\beta_{1}\right)}{\mu \beta_{1}}$ and we have $\rho_{0}<1 \Longleftrightarrow \rho<1 \Longrightarrow$ Positive Recurrent

We must now consider our L.S.T. $\mathcal{W}^{*}(s)$ :

$$
\begin{aligned}
\mathcal{W}^{*}(s)=\mathcal{G}_{N}\left(1-\frac{s}{\lambda}\right) & =\frac{K}{\left(1-\rho_{0}\left(1-\frac{s}{\lambda}\right)\right)\left(1-\rho_{1}\left(1-\frac{s}{\lambda}\right)\right)} \\
& =\frac{K \lambda^{2}}{\left(\lambda-\rho_{0}(\lambda-s)\right)\left(\lambda-\rho_{1}(\lambda-s)\right)} \\
& =\frac{K \lambda^{2}}{\left(\lambda\left(1-\rho_{0}\right)+\rho_{0} s\right)\left(\lambda\left(1-\rho_{1}\right)+\rho_{1} s\right)} \\
& =\frac{K \lambda^{2}}{\lambda^{2}\left(1-\rho_{0}\right)\left(1-\rho_{1}\right)+\lambda \rho_{1}\left(1-\rho_{0}\right) s+\lambda \rho_{0}\left(1-\rho_{1}\right) s+\rho_{0} \rho_{1} s^{2}} \\
& =\frac{K \lambda^{2}}{\lambda^{2}\left(1-\rho_{0}\right)\left(1-\rho_{1}\right)+\lambda \rho_{1} s-\lambda \rho_{0} \rho_{1} s+\lambda \rho_{0} s-\lambda \rho_{0} \rho_{1} s+\rho_{0} \rho_{1} s^{2}} \\
& =\frac{K \lambda^{2}}{\lambda^{2}\left(1-\rho_{0}\right)\left(1-\rho_{1}\right)+\lambda s\left(\rho_{0}+\rho_{1}\right)+\rho_{0} \rho_{1}\left(s^{2}-2 \lambda s\right)}
\end{aligned}
$$

We now need some substitutions: namely:

$$
\begin{aligned}
& K=\left(1-\rho_{0}\right)\left(1-\rho_{1}\right)=\frac{\beta_{1}(\mu-\lambda)-\lambda\left(\beta_{2}+\mu\right)}{\beta_{1} \mu}=\frac{\beta_{1}(\mu-\lambda)-\lambda \mu}{\beta_{1} \mu}=1-\frac{\lambda\left(\mu+\beta_{1}\right)}{\mu \beta_{1}}=1-\rho \\
& \rho_{0} \rho_{1}=\frac{\lambda^{2}}{\mu \beta_{1}} \\
& \rho_{0}+\rho_{1}=\frac{\lambda\left(\lambda+\mu+\beta_{1}+\beta_{2}+\sqrt{\left(\lambda+\mu+\beta_{1}+\beta_{2}\right)^{2}-4 \mu \beta_{1}}\right)}{2 \mu \beta_{1}}+\frac{\lambda\left(\lambda+\mu+\beta_{1}+\beta_{2}-\sqrt{\left(\lambda+\mu+\beta_{1}+\beta_{2}\right)^{2}-4 \mu \beta_{1}}\right)}{2 \mu \beta_{1}} \\
& \quad=\frac{2 \lambda\left(\lambda+\mu+\beta_{1}+\beta_{2}\right)}{2 \mu \beta_{1}}=\frac{\lambda^{2}+\lambda\left(\mu+\beta_{1}\right)}{\mu \beta_{1}}=\frac{\lambda^{2}}{\mu \beta_{1}}+\rho
\end{aligned}
$$

Proceeding where we left off, we have:

$$
\begin{aligned}
\mathcal{W}^{*}(s)=\mathcal{G}_{N}\left(1-\frac{s}{\lambda}\right) & =\frac{\lambda^{2}(1-\rho)}{\lambda^{2}(1-\rho)+\lambda s\left(\frac{\lambda^{2}}{\mu \beta_{1}}+\rho\right)+\frac{\lambda^{2}}{\mu \beta_{1}}\left(s^{2}-2 \lambda s\right)} \\
& =\frac{\lambda^{2}(1-\rho)}{\lambda^{2}(1-\rho)+\lambda s\left(\rho-\frac{\lambda^{2}}{\mu \beta_{1}}\right)+\frac{\lambda^{2}}{\mu \beta_{1}} s^{2}}
\end{aligned}
$$




$$
\begin{aligned}
& =\frac{(1-\rho) \mu \beta_{1}}{\left(\mu \beta_{1}-\lambda\left(\mu+\beta_{1}\right)+s\left(\mu+\beta_{1}-\lambda\right)+s^{2}\right)} \\
& =\frac{s(1-\rho) \mu \beta_{1}}{s\left((s+\mu)\left(s+\beta_{1}\right)-\lambda\left(\mu+\beta_{1}\right)-s \lambda\right)} \\
& =\frac{s(1-\rho) \mu \beta_{1}}{s(s+\mu)\left(s+\beta_{1}\right)-s \lambda\left(\mu+\beta_{1}\right)-s^{2} \lambda} \\
& =\frac{s(1-\rho) \mu \beta_{1}}{s(s+\mu)\left(s+\beta_{1}\right)-\lambda(s+\mu)\left(s+\beta_{1}\right)+\lambda \mu \beta_{1}} \\
& =\frac{s(1-\rho)\left(\frac{\mu}{s+\mu}\right)\left(\frac{\beta_{1}}{s+\beta_{1}}\right)}{s-\lambda+\lambda\left(\frac{\mu}{s+\mu}\right)\left(\frac{\beta_{1}}{s+\beta_{1}}\right)}
\end{aligned}
$$

Since our "waiting time" includes the customer's service time, this matches what is given on by J.W. Cohen (Cohen, 2012) on page 255 . Therefore, we conclude that the stationary queue length is equivalent in distribution to an $\mathrm{M} / \mathrm{HE} / 1$.

\subsection{Instantaneous Success / Failure}

We can also study somewhat familiar type of queue as follows:

\section{Proposition 10.4.}

If $\beta_{1} \rightarrow \infty$ with $\beta_{1}=\gamma \beta_{2}$, we have a queue with instantaneous 'success' or 'failure' after the service time has elapsed, where the probability of a successful service is $\mathrm{p}_{\mathrm{s}}=\frac{\gamma}{1+\gamma}$ and likewise for failure, we have $\mathrm{p}_{\mathrm{f}}=\frac{1}{1+\gamma}$. This queue has a stationary queue length which is equivalent to an $M / M / 1$ queue with service time $\sim$ Exponential $\left(\mu p_{s}\right)$.

Proof.

Computing the eigenvalues of $\mathbf{R}$ under these conditions yields:

$$
\begin{aligned}
& \lim _{\beta_{1}=\gamma \beta_{2} \rightarrow \infty} \rho_{0}=\lim _{\beta_{1}=\gamma \beta_{2} \rightarrow \infty} \frac{\lambda\left(\lambda+\mu+\beta_{1}+\beta_{2}+\sqrt{\left(\lambda+\mu+\beta_{1}+\beta_{2}\right)^{2}-4 \mu \beta_{1}}\right)}{2 \mu \beta_{1}}=\frac{\lambda(1+\gamma)}{\mu \gamma}=\frac{\lambda}{\mu p_{s}}=\rho \\
& \lim _{\beta_{1}=\gamma \beta_{2} \rightarrow \infty} \rho_{1}=\lim _{\substack{\beta_{1}=\gamma \beta_{2} \rightarrow \infty \\
\rho<1 \Longleftrightarrow \text { Positive Recurrent }}} \frac{\lambda\left(\lambda+\mu+\beta_{1}+\beta_{2}-\sqrt{\left(\lambda+\mu+\beta_{1}+\beta_{2}\right)^{2}-4 \mu \beta_{1}}\right)}{2 \mu \beta_{1}}=0
\end{aligned}
$$

We can now compute our P.G.F. with the appropriate substitutions. $\mathcal{G}_{\mathrm{N}}(z)=\frac{1-\rho_{0}}{1-\rho_{0} z} \frac{1-\rho_{1}}{1-\rho_{1} z}=\frac{1-\rho}{1-\rho z}$

This shows that the P.G.F. of $\mathrm{N}$ matches that of the classical M/M/1 queue with arrival rate $\mu p_{s}$, as given on page 32 of Adan, I., \& Resing, J. (Adan \& Resing, 2001), we thus conclude that the stationary queue lengths are equivalent in distribution.

\section{References}

Adan, I., \& Resing, J. (2001). Queueing Theory. Eindhoven, North Brabant, Netherlands: Eindhoven University of Technology, Dept. of Mathematics and Computing Science.

Adan, I., Wessels, J., \& Zijm, W. H. M. (1993). Matrix-geometric analysis of the shortest queue problem with threshold jockeying. Operations Research Letters, 13(2), 107-112. https://doi.org/10.1016/0167-6377(93)90037-H.

Cohen, J. W. (2012). The Single Server Queue. North-Holland Series in Applied Mathematics and Mechanics. New York, NY: Elsevier.

Guo, Pei-Chang. (2014). Newton-Shamanskii Method for a Quadratic Matrix Equation Arising in Quasi-Birth-Death Problems. East Asian Journal on Applied Mathematics. 4.4 https://doi.org/10.4208/eajam.040914.301014a

Ibe, Oliver (2011). Fundamentals of Stochastic Networks. New York, NY: John Wiley \& Sons, Inc.

Ibe, Oliver (2013). Markov Processes for Stochastic Modeling. New York, NY: Elsevier.

Keilson, J., \& Servi, L. D. (1988). A distributional form of Littles law. Operations Research Letters, 7(5), $223-227$. https://doi.org/10.1016/0167-6377(88)90035-1

Latouche, G., \& Ramaswami V. (1999). Introduction to Matrix Analytic Methods in Stochastic Modeling, 33-60. Philadelphia, PA: SIAM https://doi.org/10.1137/1.9780898719734.ch2

Little, John D. C. (1961). A Proof for the Queuing Formula: L $=\lambda W$. Operations Research Letters, 9(3), $383-387$. https://doi.org/10.1287/opre.9.3.383

Neuts, M. F. (1981). Matrix-geometric Solutions in Stochastic Models: An Algorithmic Approach. Mineola, NY: Dover Publications. 
Ross, Sheldon M. (2006). Introduction to Probability Models. Tenth Edition. Orlando, FL: Academic Press, Inc.

Seo, Sang-Hyup, Seo, Jong Hyun \& Kim, Hyun-Min (2014). Newton's method for solving a quadratic matrix equation with special coefficient matrices. Journal of Physics: Conference Series. 490.1 https://doi.org/10.1088/1742$6596 / 490 / 1 / 012001$.

$\mathrm{Xu}$, Xiuli \& Tian, Naishuo (2009). Performance Analysis of an M/M/1 Working Vacation Queue with Setup Times. Advances in Queueing Theory and Network Applications, 65-76. New York, NY: Springer https://doi.org/10.1007/9780-387-09703-9_4

\section{Copyrights}

Copyright for this article is retained by the author(s), with first publication rights granted to the journal.

This is an open-access article distributed under the terms and conditions of the Creative Commons Attribution license (http://creativecommons.org/licenses/by/4.0/). 